\title{
A RANKING MECHANISM FOR BETTER RETRIEVAL OF DATA FROM CLOUD
}

\author{
${\text { Yashasvi B } \mathbf{C}^{\mathbf{1}} \text {, R.Kanagavalli }}^{\mathbf{2}}$ \\ ${ }^{1}$ Student, Dept. of ISE., TOCE, VTU, Bangalore, Karnataka, India \\ ${ }^{2}$ Professor, Dept. of ISE., TOCE, VTU, Bangalore, Karnataka, India
}

\begin{abstract}
Most of the web services run on cloud because of the pay-as-you -go concept of cloud. Users always require the best service for any specific application. But the challenge arises in determining the best cloud computing service for a specific application. As cloud can support large number of users some situation occurs wherein the cloud service providers are not able to deliver the requested services within the requested time. In order to rank for better services $Q o S$ values are required and each user should rank for each web services but it is not time efficient as well as cost effective. In order to avoid this scenario we propose an approach which directly predicts the QoS ranking by taking past QoS data of other consumers without checking for the subsequent QoS values of other web services. An enhanced greedy algorithm is proposed to provide better accuracy.This paper also compares enhanced greedy algorithm along with greedy algorithm.
\end{abstract}

Keywords-Quality-of-service, cloud service, greedy algorithm

\section{INTRODUCTION}

Cloud computing has emerged as a concept to deliver ondemand resources such as infrastructure, platform, software, etc. to customers. The various advantages of cloud has been the reason for its major success in the information technology field .In most of the cloud computing services, QoS is an important concept and so QoS is always considered as one of the most important research topic in cloud. For every web services, any user needs to know how optimal the web services are. So, the parameter used to know how optimal a web service is the QoS parameters. The QoS values of the web services help in making the decision of how optimal any web service is as compared to a collection of web services. Quality-of Service is based on nonfunctional characteristics of Web services [1]. QoS properties are user dependent where different values provided by different users (e.g., response time, throughput, etc.). To know the optimal web service available, obtaining the user-dependent QoS values are important but obtaining of such values are not an easy task as Web services calculation for client-side requires real world invocation of web services. The invocation of real world web services is not very much advantageous as it may result to many issues. The issues include cost ineffectiveness, difficulties in efficient evaluation of services. So, optimal selection of web services is thus difficult to achieve. In order to overcome this issue, this paper proposes which directly predicts the QoS ranking by taking past QoS data of other consumers without checking for the subsequent QoS values of other web services. An enhanced greedy algorithm is proposed to provide better accuracy.

\section{PROPOSED METHOD}

In this section, we present where Quality-of-service is measured at the client side. Some of the commonly used client-side QoS properties include response time, throughput, failure probability, etc. Hence more accurate measurements provided by Client side QoS properties for the user usage experience. The Input from active user is received and Web service QoS values is processed. Based on the user provided values, similar between active users and QoS values from other users can be obtained. After that, performs similar users by taking advantage of past usage service experience for ranking purpose and later eliminates the invalid similar values for better ranking. Finally, performs enhanced greedy algorithm to order to arrange services for each users and replying back for the requested services by each users. 


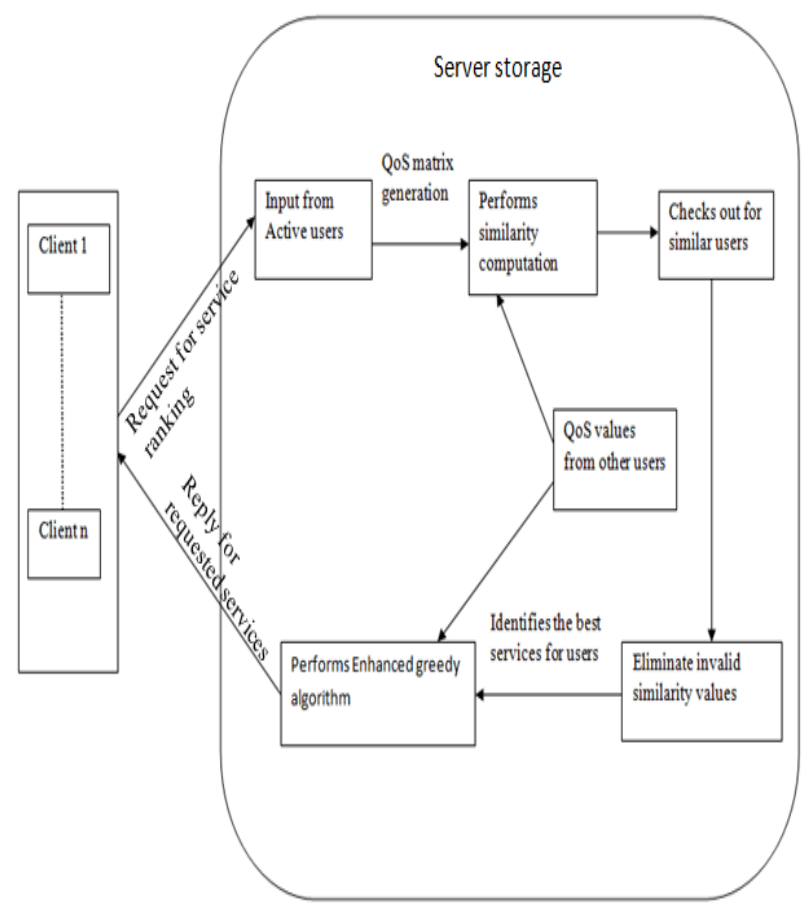

Fig .1.Proposed Architecture

\section{RELATED WORK}

Quality-of-Service (QoS) is usually employed for describing the non-functional characteristics of Web services and employed as an important differentiating point of different Web services. Based on the QoS performance of Web services, various approaches have been proposed for Web service selection [5], [6], which enables optimal Web service to be identified from a set of functionally similar or equivalent Web service candidates. To obtain the values of the user-dependent QoS properties for a certain user, Web service evaluations from the client side are usually required [7], [8], [9]. To avoid the expensive real-world Web service invocations, our work employs the information of other similar service users as well as similar Web services to predict the QoS values for the active users.

The rating-based collaborative filtering approaches try to predict the missing QoS values in the user-item matrix as accurately as possible. However, in the ranking-oriented, accurate missing value prediction may not lead to accuracy ranking prediction. Hence, ranking-oriented collaborative filtering approaches are becoming more attractive.

\section{CONCLUSIONS}

In this paper, we propose a prediction of QoS ranking for services, by taking past usage of other users which requires no additional invoking of services when ranking of QoS. Our approach is to provide best services to consumers by improving the throughput and response time.
Our future work will include in improving accuracy of ranking by using additional technique like data smoothing and matrix factorization.

\section{REFERENCES}

[1] V. Deora, J. Shao, W. Gray, and N. Fiddian, "A Quality of Service Management Framework Based on User Expectations," Proc First Int'l Conf. Service-Oriented Computing (ICSOC '03), pp. 104-114, 2003

[2] G. Wu, J. Wei, X. Qiao, and L. Li, "A Bayesian Network Based Qos Assessment Model for Web Services," Proc. IEEE Int'l Conf. Services Computing (SCC '07), pp. 498505,2007

[3] Z. Zheng and M.R. Lyu, "A Distributed Replication Strategy Evaluation and Selection Framework for Fault Tolerant Web Services," Proc. Sixth Int'l Conf. Web Services (ICWS '08), pp. 145- 152, 2008.

[4] J.L. Herlocker, J.A. Konstan, A. Borchers, and J. Riedl, "An Algorithmic Framework for Performing Collaborative Filtering," Proc. 22nd Int'l ACM SIGIR Conf. Research and Development in Information Retrieval (SIGIR '99), pp. 230-237, 1999

[5] P.A. Bonatti and P. Festa, "On Optimal Service Selection," Proc. 14th Int'l Conf. World Wide Web (WWW'04), pp. 530-538, 2005

[6] V. Cardellini, E. Casalicchio, V. Grassi, and F.L. Presti, "Flow- Based Service Selection for Web Service Composition Supporting Multiple QoS Classes," Proc. Fifth Int'l Conf. Web Services (ICWS '07), pp. 743-750, 2007.

[7] V. Deora, J. Shao, W. Gray, and N. Fiddian, "A Quality of Service Management Framework Based on User Expectations," Proc First Int'l Conf. Service-Oriented Computing (ICSOC '03), pp. 104-114, 2003

[8] E. Maximilien and M. Singh, "Conceptual Model of Web Service Reputation," ACM SIGMOD Record, vol. 31, no. 4, pp. 36-41, 2002

[9] G. Wu, J. Wei, X. Qiao, and L. Li, "A Bayesian Network Based Qos Assessment Model for Web Services," Proc. IEEE Int'l Conf. Services Computing (SCC '07), . 498505,2007

\section{BIOGRAPHIES}

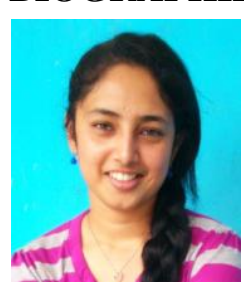

Ms. Yashasvi B C received her Bachelor of Engineering in Electronics and Communication in 2011.Currently she is a M.Tech student in Computer Networking Engineering from Visvesvaraya Technological University at The Oxford College of Engineering, Bangalore. Her research interests are Computer Networks and Network Security 